IMTC 2006 - Instrumentation and Measurement

Technology Conference

Sorrento, Italy 24-27 April 2006

\title{
Robust Sine Wave Fitting in ADC Testing
}

\author{
Attila Sárhegyi, István Kollár \\ Department of Measurement and Information Systems, \\ Budapest University of Technology and Economics, \\ H-1117 Budapest, Magyar tudósok krt.2., Hungary \\ Phone: +36 1 463-1774, Fax: +36 1 463-4112, \\ E-mail: sarhegyi@mit.bme.hu; kollar@mit.bme.hu.
}

\begin{abstract}
The IEEE standard 1241-2000 defines ADC testing methods which make use of least squares sine wave fitting algorithms. After sine fitting an error sequence is obtained and this is analyzed in order to determine the properties of the ADC. If the quantization noise dominates in this error sequence, the ADC error analysis may become somewhat misleading. This appears when the amplitude of the test signal is small enough or the amplitude is out of the range of the $A D C$.

In this paper a new method is proposed and analysed to remedy the above phenomena. Furthermore, the new algorithm is compared with dithering.
\end{abstract}

Keywords - IEEE standard 1241-2000, ADC test, sine wave fitting, three-parameter method, four-parameter method, least squares, $A D C$ test program, MATLAB.

\section{INTRODUCTION}

Numerous problems can emerge during an ADC test. One of them appears when the amplitude of the sine wave signal have to be chosen. Theoretically, the full scale excitation would be the best solution. If so, we get samples from every quantum level. It is needed in order to get a reasonable accurate parameter estimation. Adjusting a suitable amplitude is difficult and not always possible, especially when the properties of the converter are unknown. In addition, a lot of users do not know how the test should be done and apply an arbitrary amplitude between the limits, sometimes outside the limits. So, the amplitude can be changed in a wide range which affects the results of the test. On the one hand, too high amplitude results in signal degradation, on the other hand, the smaller the amplitude the more quantum levels will be missed. Moreover, if the amplitude of the input signal is too small or the quantization is too rough, the "pseudo quantization noise" model is getting to lose its validity. This means that quantization noise becomes less uniformly distributed and becomes more dependent on the input signal which results in biased estimation.

The standards [1] [2] define the way how to make a reasonable sine fit to the measured data but make no consideration of the parameters of the test signal such as the amplitude. Unfortunately, no standard can deal with all details of the associated calculation procedures. There are some features that are not necessarily included in the standard but are useful to be considered and to be included into an ADC test program. The main purpose is to prepare the further development of the standard $A D C$ test program for MATLAB [3]. This paper suggests a supplement to this test program.

In the standards [1],[2] three- and four-parameter Least Squares (LS) model fitting procedures proposed. These methods are based on an error sequence minimization and the properties of ADC's are calculated from this error sequence. The problem is that if the quantization noise dominates and the amplitude or the grain size of the quantizer is not suitable, the error sequence will be distorted and will depend on the parameters of the test signal. Consequently, the estimation may become strongly biased and will depend on the test signal. This dependence comes from the usage of a linear model fitting (3parameter LS) to a nonlinear transformation (quantization). In this paper a new method is analyzed to decrease these effects. In Section II the main properties of the applied original LS fitting are repeated. The error sequence mainly consist of the quantization noise, integral and differential nonlinearities and an additional noise. The quantization noise and pseudo quantization noise (PQN) model are analysed in Section III since deviation cause the error if the additional noise is small. Effects of the additional noise and nonlinearities are discussed in Sections V and VI. In Section IV a new "peak elimination" method is proposed to decrease the quantization noise degeneration. It is well known this kind of distortion also can be decreased by using dither. Section V summarizes some dither procedures and they are compared with the new proposed method in Section VI.

\section{THE PROPERTIES OF ORIGINAL LEAST SQUARES FIT}

In order to understand the root of the problem first of all let us examine the properties of the Original Least Squares (LS) fitting. In the standard [1], the next sum of the errors is mini- 
mized with respect to $A, B, C$, and $\omega$ :

$$
\sum_{n=1}^{M}\left(y_{n}-A \cos \left(\omega t_{n}\right)-B \sin \left(\omega t_{n}\right)-C\right)^{2},
$$

where $y_{n}$ contains the output samples of the ADC. If $\omega$ is known, the model is linear in the parameters. So, linear least squares (3-parameter method) is executed. If $\omega$ is unknown, nonlinear least squares (4-parameter method) is performed. In the latter case the method is an iterative procedure and 3parameter fitting is executed in every iterative step. Hence, only linear least squares fitting is being considered further. In matrix notation, the sum of squared differences in equation (1) is given by

$$
S=\mathbf{e}^{T} \mathbf{e}=(\mathbf{y}-\mathbf{D} \hat{\mathbf{x}})^{T}(\mathbf{y}-\mathbf{D} \hat{\mathbf{x}})
$$

where $\hat{\mathbf{y}}=\mathbf{D} \hat{\mathbf{x}}$ is the fitted model which is clearly linear in $\hat{\mathbf{x}}=\left[\begin{array}{lll}\hat{A} & \hat{B} & \hat{C}\end{array}\right]^{T}$. The estimate $\hat{\mathbf{x}}$ can be obtained from the minimum of $S=\mathbf{e}^{T} \mathbf{e}$ with respect to $\hat{\mathbf{x}}$, which is therefore

$$
\hat{\mathbf{x}}=\left[\mathbf{D}^{T} \mathbf{D}\right]^{-1} \mathbf{D}^{T} \mathbf{y}
$$

LS fitting is perhaps the most popular method for model fitting due to the simple algorithm and the nice properties especially when the error sequence is random, zero-mean Gaussian and white. If so, the estimate is unbiased, minimum variance and coincides with the maximum likelihood estimate, which is

$$
\hat{\mathbf{x}}=\left[\mathbf{D}^{T} \mathbf{R}^{-1} \mathbf{D}\right]^{-1} \mathbf{D}^{T} \mathbf{R}^{-1} \mathbf{y}
$$

where $\mathbf{R}$ is the covariance of $\mathbf{e}$ and equals to $\sigma^{2} \mathbf{I}$ if $\mathbf{e}$ fulfils the above mentioned conditions. Now, confine our attention to the estimates' mean and variance. The finite-sample bias and covariance in $\hat{\mathbf{x}}$ can be expressed by

$$
\begin{gathered}
b=\underset{\hat{\mathbf{x}}}{\mathrm{E}}[\hat{\mathbf{x}}]-\mathbf{x}=\left[\mathbf{D}^{T} \mathbf{D}\right]^{-1} \mathbf{D}^{T} \mathrm{E}[\mathbf{e}] \\
\operatorname{cov} \hat{\mathbf{x}}=\left[\mathbf{D}^{T} \mathbf{D}\right]^{-1} \mathbf{D}^{T} \mathbf{R D}\left[\mathbf{D}^{T} \mathbf{D}\right]^{-1}=\sigma^{2}\left[\mathbf{D}^{T} \mathbf{D}\right]^{-1}
\end{gathered}
$$

where $\mathrm{E}[\mathbf{e}]$ and $\sigma^{2}$ are the mean and variance of the error sequence,respectively.

The LS fit can be done for any data set and it is reasonable even if, the estimated parameters are not behave well as above. The Gaussian assumption simplifies the theory but cannot be made blindly. It can be seen from (5) that if Gaussian conditions are not met (like for quantization) the estimate may become biased and non-minimum variance.

\section{QUANTIZATION NOISE ANALYSIS}

In sine wave testing of ADC's the above mentioned Gaussian assumptions are far from being true for the error sequence.
In the "pseudo quantization noise" (PQN) model the quantization noise is treated as a random, uniformly distributed white noise between $\pm \Delta / 2$. This can be applied if Widrow's Quantization Theory II (QT II) is satisfied [4]. This means that the quantization grain size $(\Delta)$ must be made small enough, i.e. either the amplitude or the resolution of the quantizer has to be high enough. If so, the estimation still remains approximately unbiased. Since in practice no signal has a truly bandlimited characteristic function (CF) with $\pm 2 \pi / \Delta$ bandlimits, QT II is just an approximation and CF's satisfy that more or less.

Suppose that QT II is not at all satisfied and the CF is much wider then necessary and let us observe the error sequence. It

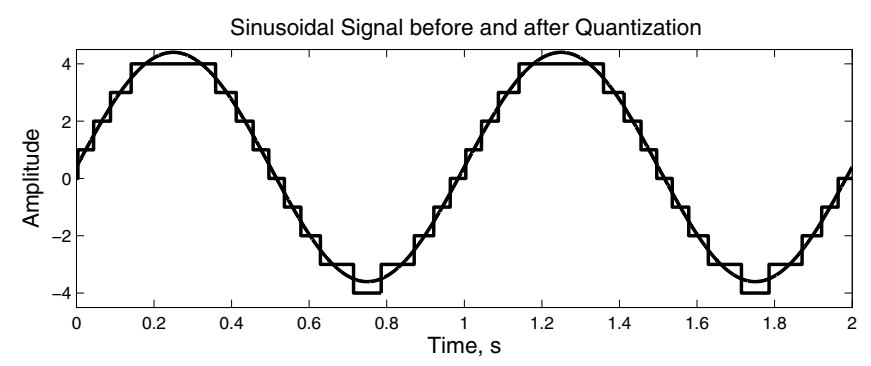

Quantization Error

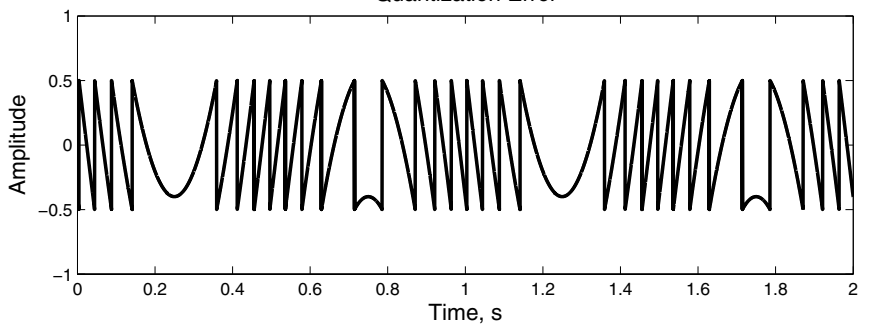

Fig. 1. The quantization error in an ideal quantizer (amplitude $=4 \Delta, \mathrm{dc}$ offset $=0.4$ and $\Delta=1$ )

can be seen in Figure 1 the quantization noise is partly deterministic and strongly depends on the input signal, especially when the sine wave cover less than, say, 50-60 quantum levels. It looks more or less like sawtooth except the sine peaks which are responsible for the strong peaks in its probability density function (PDF see Figure 2). The positions of these peaks of the PDF depend on the amplitude and the dc value. So, this PDF depends on the input signal and it is neither uniformly distributed nor zero mean which are needed for PQN model. This dependence makes the sine wave fitting worse than expected [5],[6], [7]. Small changes in the sine wave affect the residuals significantly, especially close to the peaks. ADC error analysis may become biased which is caused by the nonlinear nature of the quantization [5].

This PDF of quantization noise can be modeled as a sum of a uniformly distributed PDF and two sharp peaks. It is well known the CF can be calculated from the Fourier transform of this PDF. So, in accordance with the properties of this transformation the $\mathrm{CF}$ will be a sum of a sinc function and two smooth, not bandlimited functions. If the latter smooth functions could 


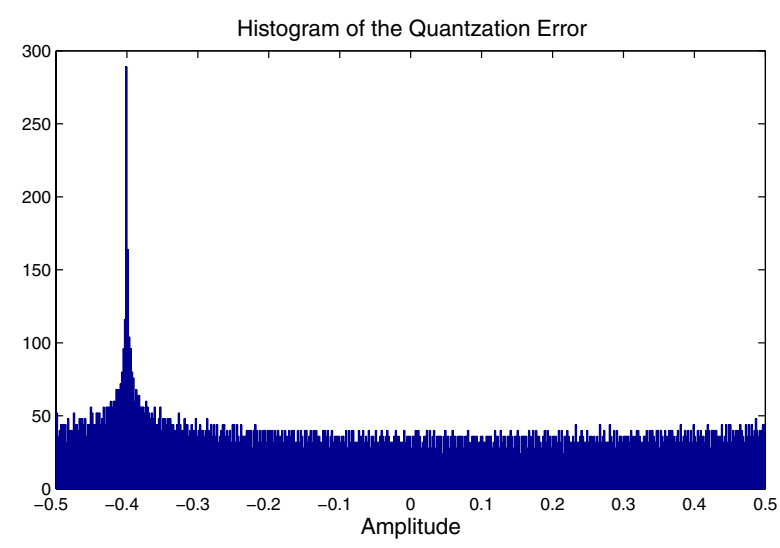

Fig. 2. Histogram of the quantization error in an ideal quantizer (amplitude $=4 \Delta$, dc offset $=0.4$ and $\Delta=1$ )

be eliminated, the PQN model would be valid again. Consequently, the PDF would not depend on the input signal and its mean value would be zero. Thus, the ADC analysis would be less biased.

After some consideration it can be seen that similar PDF can be obtained if the ADC is saturated. The main difference is that the positions of the peaks caused by the limiters do not depend on the input signal. The estimation might be biased. For instance, if the test sine has a positive dc offset and the ADC is saturated only at the positive sine peak, the mean will be biased. This can be seen in Figure 3. The peak at zero amplitude comes from saturation and the other one comes from the "peak effect" of the sine.

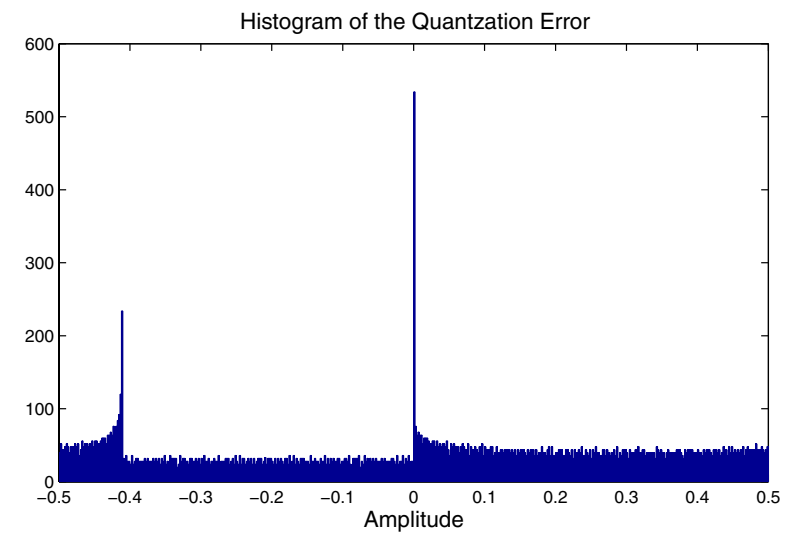

Fig. 3. Histogram of the quantization error if the ADC is saturated at the positive limit (amplitude $=3.8 \Delta$, dc offset $=0.21, \Delta=1$, limits: \pm 4 )

\section{PEAK SAMPLE ELIMINATION}

As mentioned above, the peaks in the PDF of the quantization noise are the main sources of the bias. Following the lines of [5], this effect can be decreased by eliminating the samples around the peaks of the PDF. This simply means that these samples are not used under LS fitting. Two questions appear in connection with this method.

- How many samples have to be dropped around the peaks?

- How this negligence affect for stochastical parameters?

Simulations show that the distortion is caused primarily by two peak bins. This can also be seen in Figure 1. Here are three nonlinear intervals in the quantization noise at the negative peaks of the sine, which correspond to the two lower peak bins. By eliminating these 'pathological' bins (Figure 4), QT
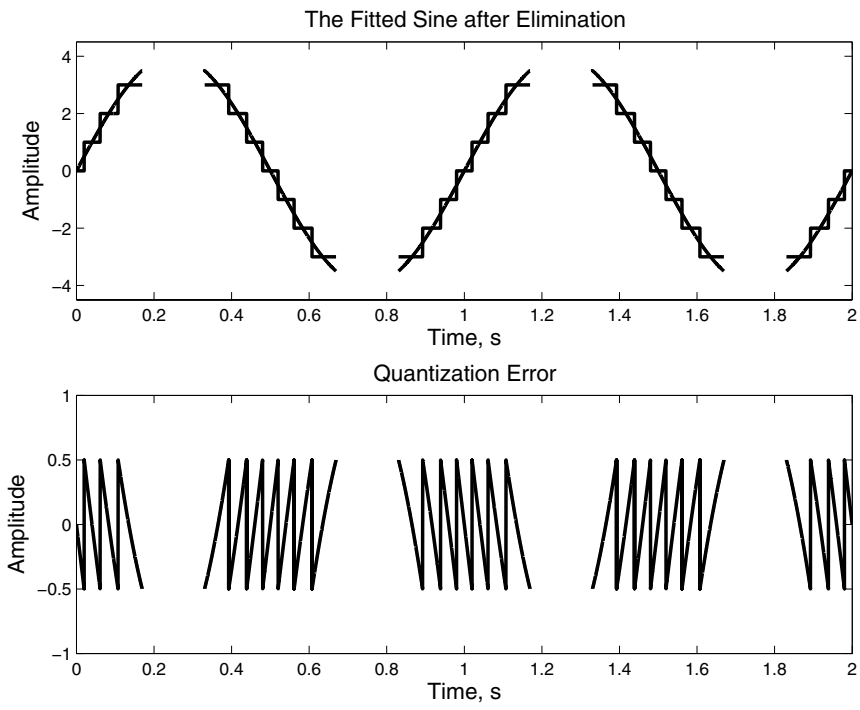

Fig. 4. The 'pathological' peaks are dropped under LS fitting

II is approximately satisfied, PDF is close to uniform distribution and the fit yields nearly unbiased amplitude estimation but the variance of the estimation increases. This is all true if there is no additional noise.

These statements can be proved by (5). Elimination of the peak samples renders the quantization noise nearly uniformly distributed (Figure 2). Hence, LS provides approximately unbiased esitmates. The variance is more difficult to determine. Suppose that the error samples are almost uncorrelated and the covariance matrix $\mathbf{R}$ is near to diagonal so, (5) is valid. $\sigma^{2}$ is the variance of the quantization noise. $\mathbf{D}^{T} \mathbf{D}$ is an $N \times N$ positive-definite matrix, at which $N$ is the number of coefficients being estimated. Its eigenvalues are positive. If a sample is dropped the eigenvalues of $\mathbf{D}^{T} \mathbf{D}$ decrease and this involves that both the inverse and its eigenvalues increase. In accordance with this intermediate result and equation (5), eliminating samples gives rise to variance increase.

Let us observe the modified algorithm if only the amplitude is unknown. For this case

$$
\left[\mathbf{D}^{T} \mathbf{D}\right]^{-1}=\frac{1}{\sum_{n=1}^{M} \cos ^{2}\left(\omega_{0} t_{n}\right)} .
$$


The variance is directly proportional to this expression, i.e. $\sigma_{A}^{2}=\sigma^{2} \cdot(1 / a)$ where $a=\sum_{n=1}^{M} \cos ^{2}\left(\omega_{0} t_{n}\right)$. The sample elimination decreases the value of $a$ by $b=\sum_{p} \cos ^{2}\left(\omega_{0} t_{p}\right)$, where $p$ denotes the peak samples. After some manipulation the new variance is

$$
\sigma_{A^{\star}}^{2}=\sigma^{2} \cdot \frac{1}{a-b}=\frac{1}{1-b / a} \cdot \sigma_{A}^{2}
$$

Here, $b / a$ represents the proportion of the "eliminated energy". After rewriting this expression to continuous time the next cost function seems acceptable for further analysis:

$$
P=\frac{b}{a}=\frac{\int_{0}^{\zeta_{c}} \cos ^{2}(x)}{\int_{0}^{\pi / 2} \cos ^{2}(x)}
$$

This cost function is evaluated only in interval $[0, \pi / 2]$, since this fraction is not changed if output signal of ADC contains an integral number of this period. If this condition is not fulfilled the value of the cost function would not be changed significantly. Before analyzing this cost function, $\zeta_{c}$ has to be derived.

In Figure 5 a cosine function ( $\mathrm{FS}=2 \mathrm{~A})$ and three code transition levels $(T[k-1], T[k], T[k+1])$ can be seen and these 'peak bins' are filled. This cosine represents the output signal of the

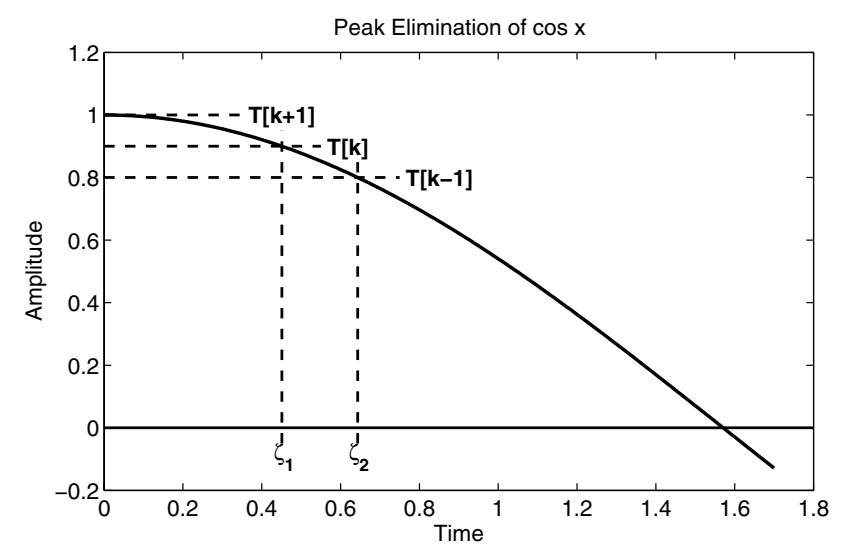

Fig. 5. Peak elimination of $\cos x$

ADC in the interval $[0, \pi / 2]$ and $\zeta_{c}$ can be derived from this function. If only one 'peak bin' is dropped, interval $\left[0, \zeta_{1}\right]$ will be eliminated under LS fitting. If two 'peak bins' are dropped, the eliminated interval will be $\left[0, \zeta_{2}\right]$. For instance, if the quantizer bit number $\mathrm{N}=8$ and $\mathrm{FS}=2 \mathrm{~A}$, the code bin width will be $\Delta=\mathrm{A} / 2^{N-1}$ and $\zeta_{1} \approx 1 / \sqrt{2^{N-2}}=1 / 8$ radians, thus the time interval $[0,1 / 8$ radians] will be eliminate in the cost function.

Hereafter, $P$ can be evaluated as a function of the quantizer bit number and of the eliminated bins (one or two bins) $P_{\zeta_{1}}[N]=$ $f_{1}, P_{\zeta_{2}}[N]=f_{2}$ (see Figure 6).

$$
f_{1}=\frac{4}{\pi} \cdot \frac{1}{\sqrt{2^{N-2}}} \quad f_{2}=\frac{4}{\pi} \cdot \frac{1}{\sqrt{2^{N-3}}}
$$

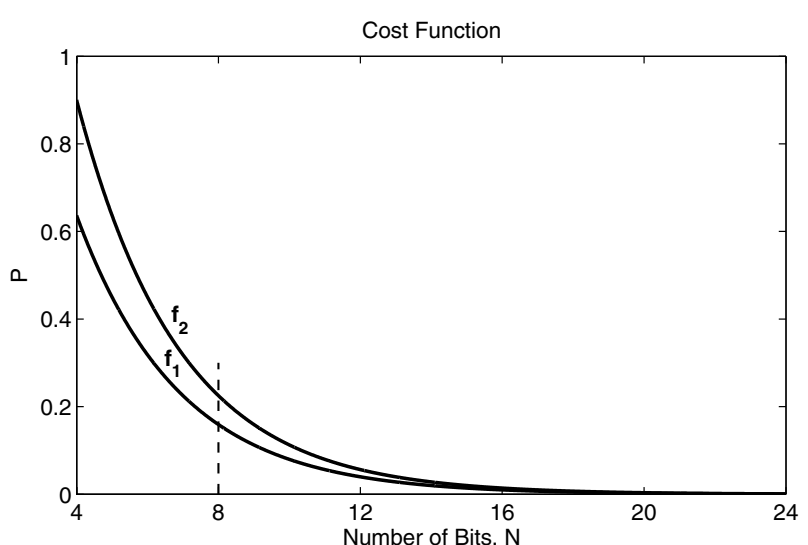

Fig. 6. The cost function as a function of the number of bits; $f_{1}$ if one peak bin eliminated, $f_{2}$ if two peak bins eliminated

Full-scale input is just a dream, but functions $f_{1}$ and $f_{2}$ give the worst case solutions if one or two 'peak bins' are dropped. For example, suppose that the peak bin is partly filled i.e. the amplitude is somewhere between two transition levels $T[k] \leq$ $A \leq T[k+1]$. In the present case, if only one peak bin is dropped the increase of $P$ will be under the $f_{1}$ function. If two ones are dropped $P$ will be under $f_{2}$. This can be seen in Figure 6.

According to the above the variance increase can be estimated by

$$
\sigma_{A^{\star}}^{2} \leq \frac{1}{1-P[N]} \cdot \sigma_{A}^{2}
$$

where $\sigma_{A^{\star}}^{2}$ denotes the variance of the modified estimation. Table I shows this variance as a function of the quantizer bit number if only one $\left(\sigma_{A 1^{\star}}^{2} / \sigma_{A}^{2}\right)$ or two $\left(\sigma_{A 2^{\star}}^{2} / \sigma_{A}^{2}\right)$ peak bins dropped. Note that sample elimination can be used for satu-

\begin{tabular}{|c|c|c|c|c|c|}
\hline $\mathrm{N}$ & 6 & 8 & 10 & 12 & 15 \\
\hline$\sigma_{A 1^{\star}}^{2} / \sigma_{A}^{2}$ & 1.4669 & 1.1893 & 1.0865 & 1.0414 & 1.0143 \\
$\sigma_{A 2^{\star}}^{2} / \sigma_{A}^{2}$ & 1.8187 & 1.2905 & 1.1268 & 1.0596 & 1.0203 \\
\hline
\end{tabular}

TABLE I

rated sines. This algorithm only drops the peak bin samples and does not care whether it contains a normal or a saturated sine peak. Of course, in that case the variance increase cannot be estimated by (10) because this is valid only for nonsaturated sine.

Thus, if the peak samples of the quantized signal (one or two peak bins) are not used under LS fitting the estimate yields a much more accurate amplitude estimation. If the sine is not saturated, the variance increase can be estimated by equation (10). 


\section{DITHER}

Use of dither is another opportunity to get a uniformly distributed PDF from Figure 2. Basically, dither is a random "noise" process added to a signal prior its quantization in order to control the statistical properties of the quantization error. If the dither is subtracted form the quantizer output, it is called Subtractive dithered (SD) system. Otherwise it is called Nonsubtractive dithered (NSD) system. Here, a new error have to be defined, which is the difference of the dithered system output and input signal $\varepsilon=y-x$ so-called total error. Our purpose is to get an independent, uniformly distributed quantization error by means of dither. If it is not possible, unbiased first and second moments is also enough where Sheppard's corrections are used.

\section{A. Subtractive Dither}

In accordance with [8], the total error induced by an SD quantizing system can be rendered uniformly distributed for arbitrary input distributions if the dither's characteristic function or CF fulfills the following condition:

$$
\mathrm{P}_{\nu}\left(\frac{k}{\Delta}\right)=0 \quad \forall k \in \mathbf{Z}_{0},
$$

Denote the dither PDF and $\mathrm{CF}$ as $\mathrm{p}_{\nu}(\nu)$ and $\mathrm{P}_{\nu}(u)$, respectively. $\mathbf{Z}_{0}$ defines the set of integers with exception of zero. Furthermore, it can be shown that the total error in an SD quantizing system is statistically independent of the system input if and only if (11) holds [8].

The simplest random process satisfying the condition in (11) is a uniform $\operatorname{PDF} \mathrm{p}_{\nu}(\nu)=\Pi_{\Delta}(\nu)$ ( $\Pi_{\Delta}$ is a uniformly distributed PDF between $\pm \Delta / 2$ and $\mathrm{p}_{\nu}$ is the PDF of the dither), whose associated characteristic function is a "sinc" function

$$
\mathrm{P}_{\nu}(u)=\operatorname{sinc}(u) \triangleq \frac{\sin (\pi \Delta u)}{\pi \Delta u} .
$$

Unfortunately, subtractive dithering is difficult to use in practical systems [9] therefore, nonsubtractive dither is frequently used.

\section{B. Nonsubtractive Dither}

In an NSD quantization system, it is not possible to render the total error either statistically independent of the system input or uniformly distributed for system inputs of arbitrary distribution [10]. However, $\mathrm{E}\left[\varepsilon^{m}\right]$ is independent of the distribution of the system input $x$ if and only if

$$
\begin{gathered}
\mathrm{G}_{\nu}^{(m)}\left(\frac{k}{\Delta}\right)=0 \quad \forall k \in \mathbf{Z}_{0} \\
\mathrm{G}_{\nu}(u) \triangleq \operatorname{sinc}(u) \mathrm{P}_{\nu}(u)
\end{gathered}
$$

where $\mathrm{G}_{\nu}^{(m)}$ denotes the $m$ th derivative of $\mathrm{G}_{\nu}, \mathrm{P}_{\nu}(u)$ is the characteristic function $(\mathrm{CF})$ of the dither. If the conditions in
(13) are satisfied then

$$
\mathrm{E}\left[\varepsilon^{m}\right]=\left(\frac{j}{2 \pi}\right)^{m} \mathrm{G}_{\nu}^{(m)}(0),
$$

which is precisely the $m$ th moment of a random process with $\mathrm{CF} \mathrm{G}_{\nu}$ and PDF $\Delta \Pi_{\Delta} \star \mathrm{p}_{\nu}$. This can be better illustrated by examples [10].

\section{Null Dither}

Begin with examination of an undithered system. The PDF of a "null dither" is $\mathrm{p}_{\nu}(\nu=\delta(\nu))$, its Fourier transform, i.e., its $\mathrm{CF}$ is equal to unity everywhere. Hence, $\mathrm{G}_{\nu}(u)=\operatorname{sinc}(u)$. No derivatives of this function vanish at nonzero multiples of $1 / \Delta$ (13); therefore, no moment of the total error (except the zeroth) will be functionally independent of the input distribution.

\section{Rectangular-PDF Nonsubtractive Dither}

Now, consider a system using dither with a simple rectangular pdf (RPDF) of 1 LSB peak-to-peak amplitude, with corresponding $\mathrm{CF} \mathrm{P}_{\nu}(u)=\operatorname{sinc}(u)$. Hence, $\mathrm{G}_{\nu}(u)=\operatorname{sinc}^{2}(u)$. The first derivative clearly satisfies the condition of (13), whereas the second derivative does not (nor do higher derivatevies). This indicates that the first moment, of the error signal is independent of the input, but its variance remans dependent. The first moment, or mean error, is zero for all inputs,indicating that the quantizer has been linearized by the use of this dither and the estimation is unbiased.

\section{Triangular-PDF Nonsubtractive Dither}

The most simple means of generating dither signals with more complicated PDF's is to simply sum two or more statistically independent RPDF random processes. Summation of statistically independent random processes convolves their PDF's which corresponds to the respective CF's. For instance, the sum of two such processes, each of 1 LSB peak-to-peak amplitude, yields a dither with a triangular PDF of 2 LSB peak-to-peak amplitude. Its $\mathrm{CF}$ is a square sinc function and $\mathrm{G}_{\nu}(u)=\operatorname{sinc}^{3}(u)$. The first and second derivatives of this function go to zero at the required places $k / \Delta$; therefore, this dither renders both the first and second moments of the total error independent of the system input. Higher derivatives do not meet the required conditions.

It is important to note that creating a uniformly distributed process of 1 LSB peak-to-peak amplitude is unrealizable. In practice, ADC's have integral and differential nonlinearities. Consequently, LSB is a "mean LSB", and an amplitude of exactly 1 LSB is not possible. The zeros of the associated sinc functions will not fall at integral multiple of $1 / \Delta$. So, the error moments will not be independent of the input signal.

Gaussian Nonsubtractive Dither

At the first glance, Gaussian dither does not seem to be a good choice since its CF is not bandlimited. Hence, it does not perfectly satisfy QT II. Following the lines in [11], it can be shown that if quantization grain size less than the standard deviation of the applied Gaussian distribution $(\Delta \leq \sigma)$ the total error will be uniformly distributed for arbitrary input signals. The main advantage of Gaussian dither is that it is not 
amplitude-sensitive. If it satisfies the $\Delta<\sigma$ condition, the total error will be uniformly distributed independently of the amplitude.

\section{CONCLUSION}

The error sequence consists of the quantization noise, integral and differential nonlinearities and an additional noise. The additional noise supposed to be a zero-mean, Gaussian white noise. The estimation bias caused by the first three nonlinear distortions. In Section III the quantization noise is investigated. Section IV and V introduced two methods to decrease the bias of the estimation.

The integral and differential nonlinearities cannot be separated from the quantization noise. But, this does not affect the analysis results significantly since the integral and differential nonlinearities are smeared and the eliminated samples are focused in the two peaks.

The effect of the additional Gaussian noise has already been discussed in Section V. If this additional noise is big enough ( $\Delta<\sigma$ ) the total error will be uniformly distributed for arbitrary quantization noise and the estimation will be unbiased. There is no need for neither sample elimination nor dither.

If the quantization noise dominates, the estimation will be biased. Both of the proposed algorithms decrease this bias. And both of them increase the variance. But sample elimination is realizable easier. There is no need for generating dither and the LS fitting can be executed on a reduced data record. This yields a remarkable acceleration if the data record is big enough. Another disadvantage of dither is that the uniformly distributed total error masks the original PDF of the quantization noise. So it masks important information about nonlinearities. This does not appear by using sample elimination.

It is important to note that again sample elimination means that these samples are not used in LS fitting, but in certain cases they need to be used. For example, 4-parameter sine fitting is an iterative procedure which needs an initial frequency. This is usually derived by FFT [12] and FFT needs the whole record.

\section{ACKNOWLEDGMENT}

This work was supported by the Hungarian Scientific Found (OTKA) TS49743

\section{REFERENCES}

[1] IEEE Standard 1241-2000, "IEEE Standard for Terminology and Test Methods for Analog-to-Digital Converters."

[2] IEEE Standard 1057-1994, "IEEE Standard Digitizing Waveform Recorders."

[3] J. Márkus, “ADC Test Data Evaluation Program for MATLAB," Home Page URL: http://www.mit.bme.hu/services/ieee/ADC-test/.
[4] B. Widrow,I. Kollár, M.C. Liu, "Statistical theory of quantization," IEEE Trans. on Instrumentation and Measurement, vol.45, pp.389-396, June 1996.

[5] I. Kollár, J.J. Blair, "Improved Determination of the Best Fitting Sine Wave in ADC Testing," IEEE Trans. on Instrumentation and Measurement, vol.54, pp.1978-1983, October 2005.

[6] J. Blair, T. Linnenbrink, "Corrected RMS error and effective number of bits for sinewave ADC tests," Computer Standards and Interfaces, vol.26, no.1, pp. 43-49, Jan. 2004.

[7] I. Kollár, "Evaluation of Sine Wave Tests of ADCs from Windowed Data," Computer Standards and Interfaces, vol. 22, pp. 261-268, 2000.

[8] L. Schuchman, "Dither signals and their effect on quantization noise," IEEE Trans. on Commun. Technol., vol. COMM-12, pp.162-165, Dec 1964.

[9] B. Widrow, I. Kollár, "Quantization Noise," Home Page URL: http://www.mit.bme.hu/books/quantization, Chapter 16: Dither, pp.389392.

[10] R.A. Wannamaker, S.P. Lipshitz, J. Vanderkooy, J.N. Wright, "A Theory of Nonsubtractive Dither," IEEE Trans. on Signal Processing, vol.48, no.2, pp. 499-516, Feb. 2000.

[11] B. Widrow, I. Kollár, "Quantization Noise," Home Page URL: http://www.mit.bme.hu/books/quantization, Chapter 5: Statistical Analysis of the Quantization Noise, pp.97-99.

[12] T.Z. Bilau, T. Megyeri, A. Sárhegyi, J. Márkus, I. Kollár, "Fourparameter fitting of sine wave testing result: iteration and convergence," Computer Standards and Interfaces, vol.26, no.1, pp. 51-56, Jan. 2004. 